\title{
LARGE GRAPHS WITH GIVEN DEGREE AND DIAMETER III
}

\author{
J.C. BERMOND, C. DELORME and G. FARHI \\ Université de Paris Sud, Orsay, bât. 490 \\ France
}

\begin{abstract}
The following problem arises in the study of interconnection networks: find graphs of given maximum degree and diameter having the maximum number of vertices. In this article we give a construction which enables us to construct graphs of given maximum degree and diameter, having a great number of vertices from small ones; in particular we obtain a class of graphs of diameter 3 , maximum degree $\Delta$ and having about $8 \Delta^{3} / 27$ vertices.
\end{abstract}

\section{INTRODUCTION}

We are interested in the $(\triangle, D)$ graph problem, one of the problems arising in telecommunications networks (or microprocessor networks). Let $G=(X, E)$ be an undirected graph with vertex set $X$ and edge set $E$. The distance between two vertices $x$ and $y$, denoted by $\delta(x, y)$, is the length of a shortest path between $x$ and $y$. The diameter $D$ of the graph $G$ is defined as $D=\max _{(x, y) \in x^{2}} \delta(x, y)$. The degree $d(x)$ of a vertex $x$ is the number of vertices adjacent to $x$ and $\Delta$ denotes the maximum degree of $G$. (For a survey on diameters see [1].)

The $(\Delta, D)$ graph problem is that of finding the maximum number of vertices $n(\Delta, D)$ of a graph with given maximum degree $\Delta$ and diameter $D$. This problem arises quite naturally in the study of interconnection networks: the vertices represent the stations (or processors), the degree of a vertex is the number of links incident at this vertex and the diameter represents the maximum number of links to be used to transmit a message. The problem seems to have been first set in the literature by Elspas [7]. Different contributions have been made in the $70^{\prime} \mathrm{s}$ and the known results were sumarized in storwick's article [10]. These results have been recently improved by Memmi and Raillard [8] and Quisquatter [9]. A simple bound on $n(\Delta, D)$ is given by Moore (see [3 or 4]): 
$\mathrm{n}(2, \mathrm{D}) \leq 2 \mathrm{D}+1$ and for $\Delta>2, \mathrm{n}(\Delta, \mathrm{D}) \leq \frac{\Delta(\Delta-1)^{\mathrm{D}}-2}{\Delta-2}$. The graphs satisfying the equality are called Moore graphs. It has been proved by different authors (see Biggs [ 3 chap.23]) that Moore graphs can exist only if $\Delta=2$ (the graphs being the $(2 \mathrm{D}+1)$ cycles) or if $D=2$ and $\Delta=3,7,57$ (for $\Delta=3$ and $\Delta=7$ there exists a unique Moore graph respectively Petersen's graph on 10 vertices, and Hoffman and Singleton's graph on 50 vertices, for $\Delta=57$ the answer is not known).

The aim of this article is to give a new construction, which gives graphs having a great number of vertices in particular for small diameters; as a corollary we obtain that $\underset{\Delta \rightarrow \infty}{\lim } \inf (\Delta, 3) \geq \frac{8}{27} \Delta^{3}$. This paper is to be considered as a companion of $[2,5,6]$ the construction is based on a new product of graphs which is studied in [2] and in [5,6] are given infinite families of graphs of given degree and diameter. In [5] it was proved that $n(\Delta, D) \geq\left(\frac{\Delta}{2}\right)^{D}$, which gives only $\frac{\Delta^{3}}{8}$ in the case of diameter 3 .

\section{DEFINITIONS}

\subsection{The * product}

Let $G=(X, E)$ and $G^{\prime}=\left(X^{\prime}, E^{\prime}\right)$ be two graphs. Take an arbitrary orientation of the edges of $G$ and let $U$ be the set of arcs. Finally, for each arc $(x, y)$ of $U$, let $f(x, y)$ be a one to one mapping from $X^{\prime}$ to $X^{\prime}$.

We define the product $G^{*} G^{\prime}$ as follows:

The vertex set of $G^{*} G^{\prime}$ is the cartesian product $x \times x^{\prime}$. A vertex $\left(x, x^{\prime}\right)$ is joined to a vertex $\left(y, y^{\prime}\right)$ in $G^{*} G^{\prime}$ if and only if

$$
\begin{aligned}
& \text { either } x=y \text { and }\left\{x^{\prime}, y^{\prime}\right\} \in E^{\prime}, \\
& \text { or }(x, y) \in U \text { and } y^{\prime}=f(x, y)\left(x^{\prime}\right) \text {. }
\end{aligned}
$$

Remark: $G^{*} G^{\prime}$ can be viewed as formed by $|x|$ copies of $G^{\prime}$ where two copies generated by the vertices $x$ and $y$ are joined if $(x, y)$ is an arc and in that case they are joined by a perfect matching depending on $(x, y)$.

Examples: (a) Let $G=K_{2},(1,2)$ being the oriented arc and let $G^{\prime}=C_{5}$. In Fig.2.1.a, we have represented $K_{2}{ }^{*} C_{5}$ where $E_{(1,2)}\left(x^{\prime}\right)=x^{\prime}$ and in Fig.2.1.b, $K_{2}{ }^{*} C_{5}$ where $f_{(1,2)}\left(x^{\prime}\right)=2 x^{\prime}$ $(\bmod .5)^{\prime}$. 


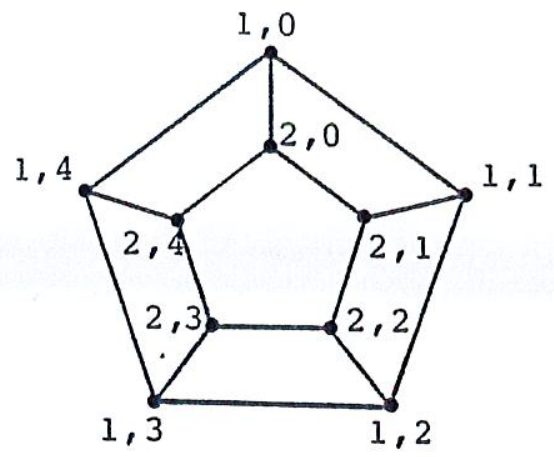

$2 \cdot 1 \cdot \mathrm{a}$

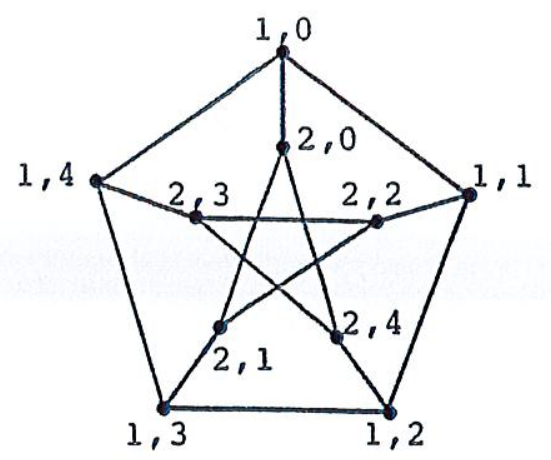

$2.1 . b$

Fig. $2.1: K_{2}{ }^{*} C_{5}$ with two different $f_{1,2}$

Note that the diameter of the graph of fig.2.1.a is 3 , but the diameter of Petersen's graph (fig.2.1.b) is 2.

(b) If we choose for any arc $(x, y) f_{X, y^{\prime}}\left(x^{\prime}\right)=x^{\prime}$ then $G^{*} G^{\prime}$ is nothing else than the cartesian sum (called also cartesian product) of $G$ and $G^{\prime}$ and in this case the diameter of $G^{\prime} G^{\prime}$ is the sum of the diameters of $G$ and $G^{\prime}$.

\section{Degrees and diameter of $G^{*} G^{\prime}$}

If $G$ is of maximum degree $\Delta$ and $G^{\prime}$ of maximum degree $\Delta^{\prime}$, then $G^{*} G^{\prime}$ has as maximum degree $\Delta+\Delta^{\prime}$. If $G$ is of diameter $D$ and $G^{\prime}$ of diameter $D^{\prime}$, then the diameter of $G^{*} G^{\prime}$ is less than or equal to $D+D^{\prime}$ and a clever choice of the functions $f_{(x, y)}$ can give a small diameter.

2.2 The property $P *$

A graph $G=(X, E)$ is said to have the property $P^{*}$ if $G$ has diameter at most 2 and if there exists an involution $f$ of $x$ (i.e. $f^{2}$ is the identity of $x$ ), such that for every vertex $x$ of $G$ we have $x=\{x\} \cup\{f(x)\} \cup\{f(\Gamma(x))\} \cup\{\Gamma(f(x))\}$.

Examples: In figure 2.2 are shown three graphs satisfying property $\mathrm{p}^{*}$ with respectively 5,8 and 9 vertices. Other examples will be given in section 4 .

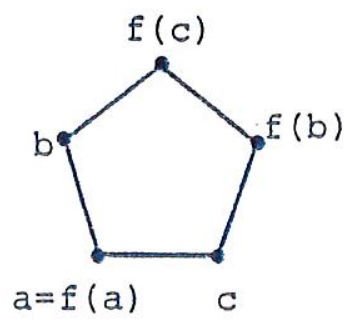

$a=f(a)$

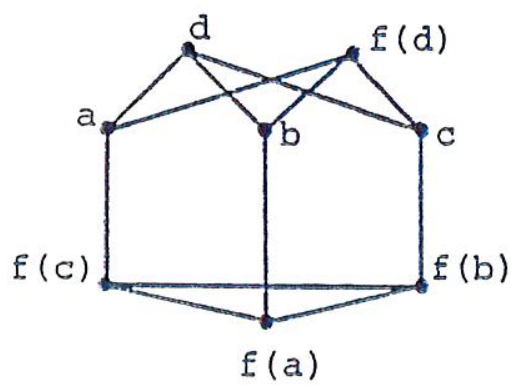

Figure 2.2

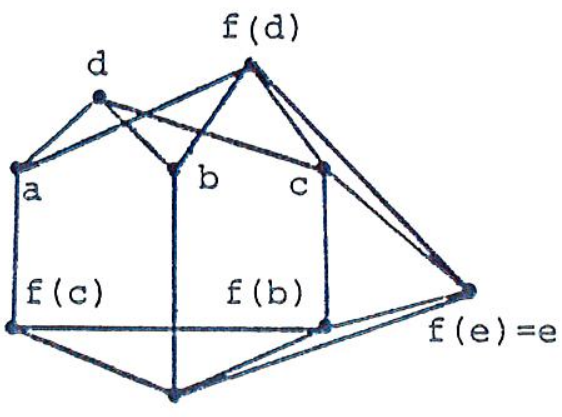




\subsection{The property $\quad$ ?}

A graph $G$ will be said to satisfy property $P$, if any pair of vertices at distance $D$ (where $D$ is the diameter of $G$ ) can be joined by a path of length $D+1$.

A trivial example is given by the complete graph $\mathrm{K}_{\mathrm{n}}, \mathrm{n} \geq 3$; other examples will be given in section 4.

\section{THE MAIN THEOREM}

Theorem: Let $G$ be a graph of diameter $D \geq 1$, satisfying property $P$ and let $G^{\prime}$ be a graph satisfying property $P^{*}$ (with involution f) - Then $G^{*} G^{\prime}$, with $f_{(x, y)}\left(x^{\prime}\right)=f\left(x^{\prime}\right)$ for every arc $(x, y)$ of an arbitrary orientation of $G$, is a graph of diameter at most Proof: Any two vertices $\left(x, x^{\prime}\right)$ and $\left(y, y^{\prime}\right)$ of $G^{*} G^{\prime}$ can be connected with a path of length at most $d_{G}(x, y)+2$ (as $G^{\prime}$ is of diameter at most 2 by property $\left.P^{*}\right)$ and therefore if $d_{G}(x, y)<D$,
then $\left(x, x^{\prime}\right)$ and $\left(y, y^{\prime}\right)$ are at distance at most $D+1$. Now let $x$ and $y$ be at distance $D$ and let $z$ be the vertex preceding $y$ on a path of length $D$ between $x$ and $y$. Let $z^{\prime}=f^{D-1}\left(x^{\prime}\right)$. Then the vertex $\left(z, z^{\prime}\right)$ is at distance $D-1$ of $\left(x, x^{\prime}\right)$; therefore the vertices in $\left(z, \Gamma\left(z^{\prime}\right)\right)$ and the vertex $\left(y, f\left(z^{\prime}\right)\right)$ are at distance at most $D$ from $\left(x, x^{\prime}\right)$ and the vertices in $\left(y, f\left(\Gamma\left(z^{\prime}\right)\right)\right)$ and $\left(y, \Gamma\left(f\left(z^{\prime}\right)\right)\right)$ at distance at most $D+1$ of $\left(x, x^{\prime}\right)$. Finally by using the path of length $D+1$ between $x$ and $y$ (by property $P$ ) the vertex $\left(y, f^{D+1}\left(x^{\prime}\right)\right)=\left(y, z^{\prime}\right)$ is at distance at most $D+1$ from $\left(x, x^{\prime}\right)$ in $G^{*} G^{\prime}$. According to the fact that by property $P *$ the vertex set of $G^{\prime}$ is equal to $\left\{z^{\prime}\right\} \cup\left\{f\left(z^{\prime}\right)\right\} \cup\left\{f\left(\Gamma\left(z^{\prime}\right)\right)\right\} U\left\{\Gamma\left(f\left(z^{\prime}\right)\right)\right\}$ every vertex of $\left(y, G^{\prime}\right)$ is at distance at most $D+1$ from $\left(x, x^{\prime}\right)$.

IV GRAPHS SATISFYING $P *$ AND $P$

\subsection{Graphs satisfying $p^{*}$}

We have seen three examples in 2.2 of degrees $2,3,4$ and respectively $5,8,9$ vertices. Note that a graph of degree $\Delta$ satisfying $p *$ has at most $2 \Delta+2$ vertices. The next construction shows the existence of graphs of degree $\Delta$ on $2 \Delta$ vertices. Proposition: For every integer $\Delta$, there exists a graph $G_{\Delta}$ satisEying property $P^{*}$, of degree $\Delta$ and having $2 \Delta$ vertices. Construction: Fig. 4.1.a shows such graphs with $\Delta=1$ and $\Delta=2$. Suppose we have constructed a graph $G_{\Delta}$, satisfying $P^{*}$, of 
degree $\Delta$ and such that the $2 \Delta$ vertices are $A$ and $f(A)$ with $|A|=|f(A)|=\Delta$ and such that $\Gamma(A) \supseteq f(A)$ and $\Gamma(f(A)) \supseteq A$ (that is satisfied by $G_{1}$ and $G_{2}$ ). Let $G_{\Delta+2}$ be the graph obtained from $G_{\Delta}$ by adding 4 vertices $x, y, f(x), f(y)$ joined between themselves and to $G$ as shown in Fig. 4.1.b. Then this graph (where the involution $f$ extends that of $\left.G_{\Delta}\right)$ has clearly degree $\Delta+2,2(\Delta+2)$ vertices and it is not difficult to show that it satisfies property $P *$ and the hypothesis of induction.

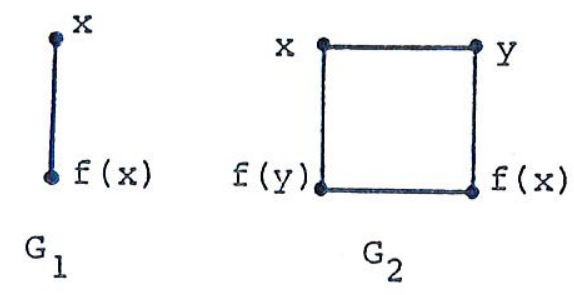

Fig. 4.1.a

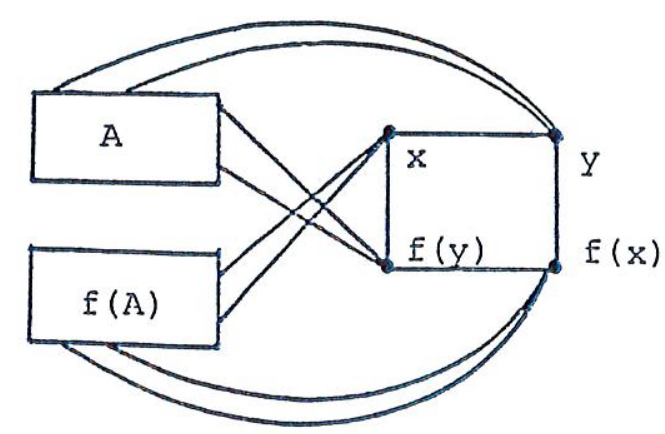

Fig. 4.1.b

\subsection{Graphs satisfying $P$}

As already noticed the complete graphs $k_{n}, n \geq 3$, satisfy $P$. One can show also that Petersen's graph, Hoffmann-Singleton's graph, the products $\mathrm{K}_{\mathrm{n}}{ }^{\mathrm{x}} \mathrm{x}, \mathrm{n} \geq 3$, where $\mathrm{x}$ is one of the graphs of Fig.2.2, satisfy property $\mathrm{P}$.

An important class of graphs (which will be used in section 5) is formed by the graphs $\mathrm{P}_{\mathrm{q}}$ of diameter 2 associated to projective planes. Let us recall, that if $q=p^{r}$ where $p$ is a prime number, there exists a projective plane of order $q$ with a polarity, that is a numbering of the points $M_{i}, 1 \leq i \leq q^{2}+q+1$ and of the lines, $D_{j}, I \leq j \leq q^{2}+q+1$ such that if $M_{j} \in D_{j}$ then $M_{j} \in D_{i}$. Thus $\underset{j \in D_{i}}{U} D_{j}$ contains all the points of the plane.

Let $P_{q}$ be the graph whose vertices are the points of the projective plane, the vertices $M_{i}$ and $M_{j}$ are joined if and only if $M_{j} \in D_{i}$. The properties of the numbering shows that $P_{q}$ is of degree $q+1$; i.t has $q^{2}+q+1$ vertices and its diameter is 2 . Now, let $M_{i}$ and $M_{j}$ be two vertices at distance 2 , then $M_{j} \leftarrow D_{i}$. As $\left|D_{i} \cap D_{j}\right|=1$, there exists a vertex $M_{k}$, such that $M_{k} \in D_{i}$ and $M_{k} ; D_{j} ;$ as $M_{k}$ is at distance $I$ from $M_{i}$ and 2 from $M_{j}$, it is clear that $M_{i}$ and $M_{j}$ are joined by a path of length 3 . Similarly one can prove that the quotient of Levi graphs of 
generalized quadrangles and hexagons, having a polarity, by that polarity have property $P$. (See [5].)

\section{APPLICATIONS}

Let $G=K_{n}$ and $G^{\prime}=x_{8}$ the graph on 8 vertices of degree 3 (see Fig. 2.2) then $K_{n} * X_{8}$ has $8 n$ vertices, degree $n+2$ and diameter 2. In particular with $\mathrm{n}=3$ (resp.4) we obtain graphs of diameter 2 , degree 5 (resp.6) on 24 (resp.32) vertices. The better graph of diameter 2 degree 6 , known before had only 31 vertices (namely $\mathrm{P}_{5}$ ) . By taking $\mathrm{K}_{3 *} \mathrm{X}_{8}$ as graph $\mathrm{G}$ and again $\mathrm{X}_{8}$ as $\mathrm{G}^{\prime}$ we obtain a graph of diameter 3 , degree 8, on 192 vertices (the better graph known before has 114 vertices). By taking for $G$ the graph on 585 vertices of degree 9 and diameter 3 and for $G^{\prime}$ the graphs on $5(8,9)$ vertices of Fig.2.1 we give examples of graphs of diameter 4 of degree 11 (resp. 12,13) on 2925 (resp. 4680, 5265) vertices which are the best known now. Another application is the following result.

Theorem: $\lim _{\Delta \rightarrow \infty} \inf n(\Delta, 3) \geq \frac{8}{27} \Delta^{3}$

Proof: Let $G$ be the graph $P_{q}$ defined in section 4, with $q$ odd, $q$ a prime power, of diameter 2 , degree $q+1$ and on $\mathrm{q}^{2}+\mathrm{q}+1$ vertices which satisfies property $P$. Let $\Delta=\frac{3(g+1)}{2}$ and let $G^{\prime}=G_{\Delta / 3}$ be the graph of degree $\frac{\Delta}{3}$ on $\frac{2 \Delta}{3}$ vertices satisfying property $P^{*}$ constructed in 4.1 . By the main theorem ${ }^{P}{ }^{*}{ }^{*} G_{\Delta / 3}$ is a graph of diameter 3 degree $q+1+\frac{\Delta}{3}=\Delta$, having $\left(q^{2}+q+1\right) \frac{2 \Delta}{3}=\left(4 \frac{\Delta^{2}}{9}-\frac{2 \Delta}{3}+1\right) \frac{2 \Delta}{3}=\frac{8 \Delta^{3}}{27}-4 \frac{\Delta^{2}}{9}+2 \frac{\Delta}{3}$ vertices proving therefore the theorem.

VI GENERALIZATIONS

\subsection{The property $\mathrm{P}_{i}$}

A graph $G$ is said to have property $P_{i}$, if there exists a permutation $f$ of its vertices, such that $f^{2}$ is an automorphism of $G$ and that the graph $G^{*}$, which has the same vertices as $G$ and whose edges are the edges of $G$ or their images by $f$, has diameter i.

\subsection{Examples}

The graph $G$ of Fig.6, on 4 vertices, where $f(x)=x+1$ (modulo 4) 
has property $\mathrm{P}_{1}, \mathrm{G}^{*}$ being the complete graph $\mathrm{K}_{4} \cdot$

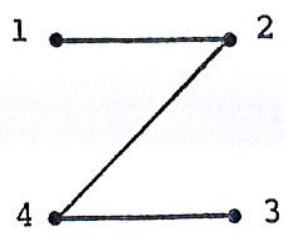

Fig. 6

The graph $G$, on 29 vertices, where the vertex $x$ and $y$ are joined if $y-x \equiv 1,-1,7,-7(\bmod .29)$ and where $f(x) \equiv 12 x$ (mod.29) has property $\mathrm{P}_{2}$.

If $4 \mathrm{e}+1$ is a prime power, consider the graph whose $4 \mathrm{e}+1$ vertices are the elements of the field $\mathrm{GF}_{4 \mathrm{e}+1}$; two vertices $x$ and $y$ are adjacent if $x-y$ is a square in $G_{4 e+1}$. This graph has property $\mathrm{P}_{1}$ (diameter 2 and degree $2 \mathrm{e}$ ). (These graphs are,known as Paley's graphs and are examples of strongly regular graphs.)

\subsection{Theorem}

Let $G$ be a graph of diameter $D \geq i+1$ and let $G^{\prime}$ be a graph satisfying property $P_{i}$. Then $G^{*} G^{\prime}$, with $f_{(x, y)}\left(x^{\prime}\right)=f\left(x^{\prime}\right)$ for every arc $(x, y)$ of an arbitrary orientation of $G$, has diameter $D+i$.

Proof: Let $x$ and $y$ be two vertices of $G$ joined by a path $P$ (not necessarily elementary) of length $j$. If this path contains a arcs of $G$ and therefore $j-a$ arcs of $-G$, let $\varepsilon(P)=2 a-j$. We will prove that if there exists a path of length $j^{\prime}$ from $f^{\varepsilon(P)}\left(x^{\prime}\right)$ to $y^{\prime}$ in $G^{\prime^{*}}$, with $j^{\prime} \leq j$, then there exists a path of length $j+j^{\prime}$ in $G^{*} G^{\prime}$ between $\left(x, x^{\prime}\right)$ and $\left(y, y^{\prime}\right)$. The proof goes by induction on $j$ (that is true for $j=0$ ) and for $j$ fixed by induction on $j^{\prime}\left(j^{\prime} \leq j\right)$; that is true for $j^{\prime}=0\left(y, f^{\varepsilon(P)}\left(x^{\prime}\right)\right)$ being joined to $\left(x, x^{\prime}\right)$ by the obvious path of length $j$. Suppose $j^{\prime}>0$ and let $\left(z^{\prime}, y^{\prime}\right)$ be the last edge of the path between $f^{E(P)}\left(x^{\prime}\right)$ and $y^{\prime}$ in $G^{\prime^{*}}$. If $\left(z^{\prime}, y^{\prime}\right) \in G^{\prime}$, we obtain a path of length $\left(j+j^{\prime}\right)$ from $\left(x, x^{\prime}\right)$ to $\left(y, y^{\prime}\right)$ by adding the arc

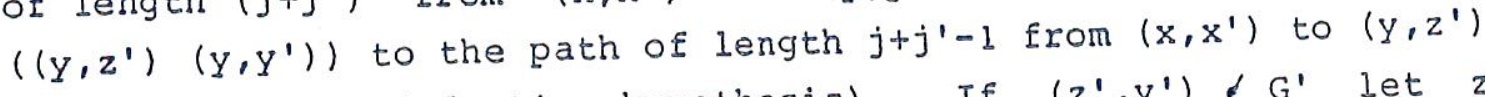
(which exists by induction hypothesis). If $\left(z^{\prime}, y^{\prime}\right) \& G^{\prime}$ let $z$ be the vertex preceding $y$ on the path $P$ of $G$; then $f^{\varepsilon(y, z)}\left(z^{\prime}\right)$ and $f^{\varepsilon(y, z)}\left(y^{\prime}\right)$ are joined by an edge in $G^{\prime}$ (recall that $\varepsilon(y, z)$ equals 1 or -1 according $(y, z)$ belongs or not to G). 
By induction hypothesis with $j-1$ and $j^{\prime-1}\left(j^{\prime}-1 \leq j-1\right)$ there exists in $G^{*} G^{\prime}$ a path of length $j^{\prime}+j^{\prime}-2$ from $\left(x, x^{\prime}\right)$ to $G^{*} G^{\prime}\left(z, f^{\varepsilon}(y, z)\right.$ and then by adding the path of length 2 of $\begin{array}{ll}G^{*} G^{\prime}\left(z, f^{\varepsilon}(y, z)\right. & \left.\left(z^{\prime}\right)\right)\left(z, f^{\varepsilon(y, z)}\left(y^{\prime}\right)\right)\left(y, y^{\prime}\right) \text { we obtain a path of } \\ \text { length } j+j^{\prime} \text { from }\left(x, x^{\prime}\right) \text { to }\left(y, y^{\prime}\right) \text {. }\end{array}$

Now let $\left(x, x^{\prime}\right)$ and $\left(y, y^{\prime}\right)$ be any two vertices of $G^{*} G^{\prime}$. There is a (not necessarily elementary) path $P$ from $x$ to $y$ of length $j=D$ or $D-1$. Since $D \geq i+1, j \geq i$ holds. As $G^{\prime *}$ is We have $j^{\prime} \leq j$ (as $j^{\prime} \leq i$, and $i \leq j$ ) and by the property proved above there exists a path of length $j+j^{\prime} \leq D+i$ from $\left(x, x^{\prime}\right)$ to $\left(y, y^{\prime}\right)$.

\subsection{Applications}

By using the example of 6.2, we can build new good graphs. For example with the Paley's graphs we obtain infinitely many graphs of diameter 3 which have actually the highest known number of vertices, although the asymptotic value obtained in theorem of section 5 is not improved. By taking for $G$ the graph of diameter 3, degree 9 on 585 vertices and for G' the Paley's graph on 13 vertices we obtain a graph of diameter 4, degree 15 on 7605 vertices. (This is. in fact the best known value).

Remark: The condition on $G, D \geq i+1$ can be deleted if one adds extra conditions on $G^{\prime}$; for example if $G$ is of diameter 1 and $G^{\prime}$ satisfies $P_{1}$ and is of diameter 2 , then $G^{*} G^{\prime}$ has diameter 2 .

\section{REFERENCES}

[1] J.C.Bermond and B.Bollobás, Diameters in graphs: a survey, Proc. 12th Southeastern Conference on Combinatorics, Graph

[2] J.C. Bermond, C. Delorme and G. Farhi, Large graphs with given
degree and diameter II, to appear.

[3] N. Biggs, Algebraic graph theory, Cambridge University Press,
Cambridge, England, 1974 .

[4] B. Bollobás, Extremal graph theory, London Math. Soc.

dernic Press, London 1978.

[6] C. Delorme, Grands graphes de degré et diamètre donnés, to appear. C. Delorme and G. Farhi, Large graphs with given degree and
diameter I, to appear.

[7] B. Elspas, Topological constraints on interconnection limited logic, Proc. 5th Symposium on switching circuit theory and logical design, I.E.E.E. 5-164 (1964), 133-197. 
[8] G. Mernmi and Y. Raillard, Some new results about the $(d, k)$ graph problem, I.E.E.E. Trans. on Computers, to appear.

[9] J.J. Quisquatter: manuscript to be published.

[10] R.M. Storwick, Improved constructions techniques for $(d, k)$ graphs, I.E.E.E. Trans. Computers, 19, (1970) 1214-1216. 